\title{
Detection and severity grading of COPD exacerbations using the exacerbations of chronic pulmonary disease tool (EXACT)
}

\author{
Alex J. Mackay, Gavin C. Donaldson, Anant R.C. Patel, Richa Singh, \\ Beverly Kowlessar and Jadwiga A. Wedzicha
}

Affiliations: Centre for Respiratory Medicine, University College London, London, UK.

Correspondence: A.J. Mackay, Centre for Respiratory Medicine, University College London, Royal Free Campus, Rowland Hill Street, London NW3 2PF, UK. E-mail: alexander.mackayaucl.ac.uk

ABSTRACT Uncertainty exists over the ability of the exacerbations of chronic pulmonary disease tool (EXACT) patient-reported outcome diary to quantify exacerbation severity and frequency. To clarify this, we investigated the ability of the EXACT to assess severity of exacerbations and examined the relationship between exacerbations diagnosed using London chronic obstructive pulmonary disease (COPD) cohort diary cards, physician review and symptom-defined events using the EXACT.

58 patients enrolled in the London Chronic Obstructive Pulmonary Disease (COPD) cohort prospectively completed the EXACT during 128 cohort diary card-defined exacerbations between January 2010 and April 2012.

Mean \pm SD EXACT scores increased from $42.6 \pm 8.6$ at baseline to $48.0 \pm 8.6$ at exacerbation onset $(\mathrm{p}<0.001)$, and rose further to a maximum score of $54.1 \pm 8.9$. Maximum EXACT scores were significantly higher in treated than untreated events. Time taken for EXACT scores to return to baseline was significantly related to symptom recovery time as judged by London COPD cohort diary cards, and to peak expiratory flow rate recovery. $\sim 50 \%$ of both diary card-defined and healthcare utilisation exacerbations crossed the EXACT event threshold. However, only $27.9 \%$ of diary card-defined and $34.6 \%$ of healthcare utilisation exacerbations fully met the criteria for an EXACT event. Patients exhibited smaller rises in the EXACT score at exacerbation as baseline disease severity increased.

The EXACT is an effective method of evaluating chronic obstructive pulmonary disease exacerbation severity. However, concerns remain about the ability of the EXACT to accurately detect exacerbations.

@ERSpublications

The EXACT is an effective method to assess exacerbation severity but uncertainty remains over its ability to detect exacerbations http://ow.ly/rHg1P

For editorial comments see page 662 .

This article has supplementary material available from www.erj.ersjournals.com

Received: June 282013 | Accepted after revision: Aug 072013 | First published online: Aug 292013

Support statement: The London COPD Cohort is funded by the MRC Patient Cohort Research Initiative (grant number G0800570/1).

Conflict of interest: Disclosures can be found alongside the online version of this article at www.erj.ersjournals.com

Copyright @ERS 2014 


\section{Introduction}

Chronic obstructive pulmonary disease (COPD) is associated with episodes of symptomatic deterioration termed exacerbations [1]. COPD exacerbations are amongst the commonest causes of medical admission to hospital [2]. Patients with frequent exacerbations [3] have accelerated lung function decline [4, 5], worse quality of life [6], are at increased risk of cardiovascular events [7] and have greater mortality [8].

Assessment of exacerbation severity is an important outcome measure in COPD and most clinical trials of preventive therapy to date have used a dichotomous approach to assigning severity levels, with outpatient drug therapy (antibiotics and/or steroids) categorised as "moderate" and hospitalisations labelled "severe". These outcome measures are limited by inaccurate reporting, lack of generalisability across different healthcare systems and their failure to capture unreported exacerbations. Unreported exacerbations are common $[6,9,10]$ and important events, associated with worsening quality of life $[6,9]$ and increased risk of subsequent hospitalisation [11].

Exacerbation symptoms systematically recorded on daily diary cards accurately detect both reported and unreported exacerbations $[6,12]$, but most symptom diary cards are not sensitive enough to assess severity of these events. The Food and Drug Administration's patient-reported outcome (PRO) guidance document [13] offers recommendations for developing PRO instruments for use in medical product development, including symptom-based methods for standardising the severity of reported and unreported exacerbations.

The exacerbations of chronic pulmonary disease tool (EXACT) is a PRO daily symptom diary developed to capture frequency, severity and duration of exacerbations in clinical trials of COPD [14]. Scores range from 0 to 100 , with higher scores indicating more severe symptoms. To date, no published data have examined the relationship between EXACT scores and pulmonary function or markers of inflammation during exacerbations. Furthermore, the EXACT was designed to quantify the evaluation of exacerbations of COPD. Specifically, the intent was to capture unreported events, using a threshold-based definition of sustained symptomatic worsening, and provide a standardised metric for evaluating the severity of unreported and reported, healthcare utilisation (HCU) events. However, in published papers to date, no data has been reported examining the relationship between symptom-defined events captured by the EXACT, exacerbations detected by London Chronic Obstructive Pulmonary Disease (COPD) cohort symptom diary cards, and HCU events.

We hypothesised that the EXACT can accurately assess the severity of both reported and unreported COPD exacerbations, defined by the London COPD cohort diary card, as measured by exacerbation length, lung function impairment and systemic inflammation. Furthermore, we sought to examine the relationship between exacerbations diagnosed using validated London COPD cohort diary cards $[4,6,12]$ and physician review, and symptom-defined events captured by the EXACT. Therefore, we prospectively administered the EXACT to patients in a well characterised COPD cohort.

\section{Methods}

\section{Patient recruitment}

This study involved 58 COPD patients enrolled in the London COPD cohort between January 2010 and April 2012. At annual review or recruitment between April 1, 2009 and May 26, 2012 a full medical history and examination was obtained. Patients were included if the post-bronchodilator forced expiratory volume in $1 \mathrm{~s}$ (FEV1) was $\leqslant 80 \%$ predicted from age, height and sex, and FEV1/forced vital capacity (FVC) ratio was $<0.7$ [15]. Patients with a history of any other significant respiratory diseases were excluded, as were those unable to complete daily diary cards.

Ethical approval for the study was granted from the Royal Free Hospital research ethics committee, London, UK, and all patients gave written informed consent. Permission to use the EXACT questionnaire was obtained from United BioSource Company (UBC, Bethesda, MD, USA).

\section{Monitoring and definition of exacerbations}

Patients were asked to record daily peak expiratory flow rate (PEFR) and any increase in respiratory symptoms on London COPD cohort diary cards. An exacerbation was defined as an increase in respiratory symptoms for two consecutive days, with at least one major symptom (dyspnoea, sputum purulence or sputum volume) plus either another major or a minor symptom (wheeze, cold, sore throat and cough), the first of which was defined as the day of onset of the exacerbation. Symptom counts were obtained by summating each increased respiratory symptom recorded on diary cards per day [12].

Exacerbation duration was defined as the number of days after onset that worsening symptoms persisted. The last day of recorded worsening symptoms before two consecutive symptom-free days defined the end of the exacerbation. Exacerbation recovery was not determinable if patients failed to record diary card 
symptoms or continuously recorded symptoms for $>99$ days after onset. Exacerbation frequency was calculated for each patient using diary card data obtained between April 1, 2010 and April 1, 2012. For recently recruited patients with $<1$ year of diary data, exacerbation frequency was based on the number of exacerbations the patient recalled for the year prior to recruitment (see online supplementary material) [16].

\section{Exacerbation assessment}

Exacerbations were treated according to the prevailing guidelines and clinical judgment with increased inhaled therapy, antibiotics and/or oral steroids. Neither the magnitude of exacerbation EXACT score nor the diary card symptom score played any role in treatment decisions. When patients attended for an exacerbation, venous blood samples were taken and spirometry performed prior to commencing exacerbation treatment. Serum C-reactive protein (CRP) was measured using Modular Analytics E 170 Module (Roche, Burgess Hill, UK).

\section{EXACT administration}

Patients completed a paper version of the EXACT at least once under supervision in the clinic and were instructed to complete the EXACT diary each evening before bedtime, based on their symptoms experienced that day. Patients prospectively completed the EXACT on a daily basis when stable and continued for the long term to enable capture of the exacerbation prodrome, the onset of the event, its nadir and recovery. Patients in the analysis completed at least one EXACT at both the symptomatic onset of an exacerbation and during a baseline period -14 to -8 days before onset. The median number of EXACT questionnaires completed per person was 196 (interquartile range 106-311). Each patient's baseline value was represented by the mean value recorded during the stable state (median 7 days (interquartile range 77 days) of data recorded during baseline period). As specified in the user manual, EXACT events were defined as a 12-point increase above baseline for two consecutive days or nine-point increase for 3 days. For the purposes of this study, we defined EXACT recovery as the time taken from exacerbation onset for the EXACT score to return to baseline value.

\section{Statistical analysis}

Data were analysed with STATA 8.2 (Stata Corporation, College Station, TX, USA). Normally distributed data were expressed as mean $\pm \mathrm{SD}$ and skewed data as median (interquartile range). Comparisons were made by paired t-test or Wilcoxon signed-rank test. The relationship between exacerbation frequency, determined using London COPD cohort diary cards, and baseline EXACT scores was examined with a negative binomial regression model, whilst Poisson regression was used to model exacerbation recovery and EXACT scores. Cross-sectional regression models were used to analyse the relationship between CRP levels during exacerbation and EXACT score as allowance could be made for repeated measures on the same patient. Total net EXACT recovery score of an exacerbation was created to give a novel measure of exacerbation severity which reflects the overall symptomatic intensity of an exacerbation. Total net EXACT recovery score of an exacerbation was calculated as the sum of daily mean change from baseline EXACT scores from exacerbation onset to symptomatic resolution as judged by London COPD cohort diary cards (online supplementary fig. S1).

\section{Results}

\section{Patient characteristics}

Full baseline clinical characteristics of the 58 patients included in this analysis are reported in table 1, which demonstrates that patients had moderate to severe COPD with a mean FEV1 of $48.6 \%$ predicted.

\section{Baseline studies}

Baseline EXACT scores were significantly related to disease severity. The baseline EXACT scores in 58 patients were significantly correlated with London COPD cohort diary card exacerbation frequency $(\rho=0.38 ; p=0.003)$, FEV $1_{1}(\rho=-0.32 ; p=0.015)$ and FEV1 $\%$ predicted $(\rho=-0.30 ; p=0.020)$.

\section{Exacerbation studies}

58 patients completed the EXACT during 128 London COPD cohort diary card exacerbations. 85 (66.4\%) out of 128 were treated with systemic therapy, $82(64.1 \%)$ with antibiotics and $64(50.0 \%)$ with oral corticosteroids. There were no hospitalised exacerbations.

The baseline mean \pm sD EXACT score averaged over 7 days ( -14 to -8 days before exacerbation onset) was 42.6 \pm 8.6. This increased to $48.0 \pm 8.6$ at London COPD cohort diary card exacerbation onset, $5.4 \pm 7.1$ above baseline $(\mathrm{p}<0.001)$, representing a $13 \%$ rise. EXACT scores increased further to a maximum score of $54.1 \pm 8.9$ or $11.4 \pm 7.6$ above baseline $(\mathrm{p}<0.001)$, during the 2 weeks following exacerbation onset (fig. 1$)$. 
TABLE 1 Stable state clinical characteristics of the 58 patients at the time of recruitment to the cohort

$\begin{array}{lr}\text { Age years } & 70.2 \pm 8.2 \\ \text { FEV1 L } & 1.22 \pm 0.56 \\ \text { FEV1 \% predicted } & 48.6 \pm 18.1 \\ \text { FEV1/FVC \% } & 46.6 \pm 14.2 \\ \text { Smoking pack-years } & 54.0 \pm 42.1 \\ \mathrm{SpO}_{2} \% & 94.6 \pm 2.0 \\ \mathrm{BMI} \text { kg·m } & \pm \\ \text { London COPD cohort diary card exacerbation frequency per patient per year } & 26.8 \pm 5.7 \\ \text { Male } & 2.88(1.92-4.43) \\ \text { Current smokers } & 36(62.1) \\ \text { Comorbidity present } & 11(19.0) \\ \text { Congestive heart failure } & 47(81.0) \\ \text { Ischaemic heart disease } & 6(10.3) \\ \text { Hypertension } & 13(22.4) \\ \text { Diabetes mellitus } & 32(55.2) \\ \text { Patients receiving maintenance inhaled corticosteroids } & 4(6.9)\end{array}$

Data are presented as mean $\pm \mathrm{SD}$, median (interquartile range) or $\mathrm{n}(\%)$. $\mathrm{FEV} 1$ : forced expiratory volume in $1 \mathrm{~s}$; FVC: forced vital capacity; $\mathrm{SpO}_{2}$ : arterial oxygen saturation measured by pulse oximetry; $\mathrm{BMI}$ : body mass index; COPD: chronic obstructive pulmonary disease.

The median time from exacerbation onset to peak EXACT score was 3 days (interquartile range 1-5.5 days). No difference was seen in the maximum increase from onset in EXACT score between type 1 Anthonisen exacerbations (events associated with increased sputum volume, purulence and dyspnoea) and other types $(\mathrm{n}=27,6.6 \pm 6.3$ versus $\mathrm{n}=101,5.9 \pm 5.4 ; \mathrm{p}=0.541)$.

\section{Relationship between EXACT score and systemic inflammation}

EXACT score changes at exacerbation presentation were significantly related to concurrent levels of systemic inflammation. At exacerbation, $\log _{10}$ CRP was related to the change in EXACT score from baseline to exacerbation $(\rho=0.30 ; p=0.041)$ (fig. 2 ). The median (interquartile range) exacerbation CRP score was 9 (4.3-16.8).

\section{Evolution of EXACT scores during exacerbation recovery}

EXACT scores accurately reflected exacerbation recovery. The time taken for EXACT scores to return to baseline (median 7 days (interquartile range $0-12$ days), $n=93$ ) was significantly related $(\rho=0.44 ; \mathrm{p}<0.001)$ to symptom recovery time as judged by London COPD cohort diary cards (median 8 days (interquartile range 4-12 days), $\mathrm{n}=114$ ). Recovery in EXACT was also significantly correlated with lung function recovery as measured by PEFR $(\rho=0.32 ; p=0.003)$. Time course plots of recovery of EXACT score, London COPD

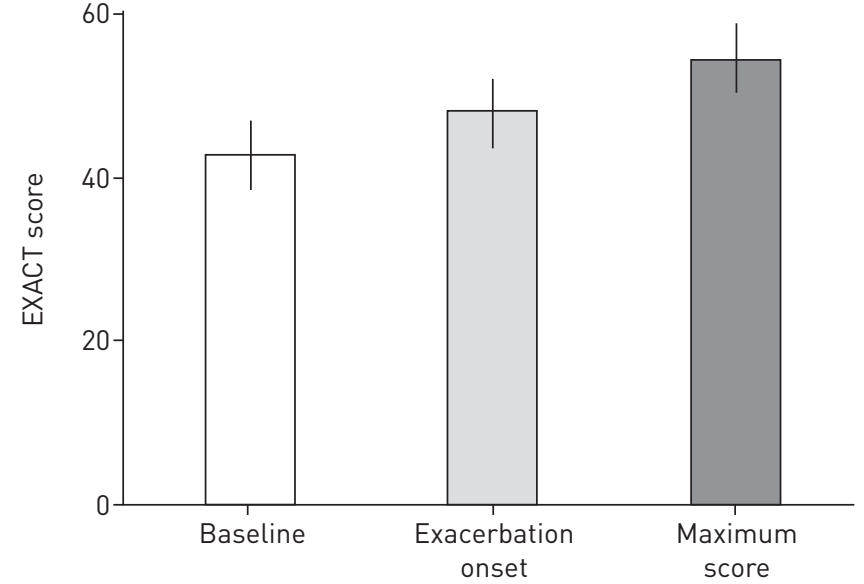

FIGURE 1 Mean exacerbations of chronic pulmonary disease tool (EXACT) scores at baseline, exacerbation onset and the maximum during the 2 weeks following exacerbation onset. Vertical lines represent standard errors. 
FIGURE 2 Relationship between $\log _{10}$ $\mathrm{C}$-reactive protein (CRP) and change in exacerbations of chronic pulmonary disease tool (EXACT) scores at exacerbation $(\rho=0.30 ; p=0.041)$.

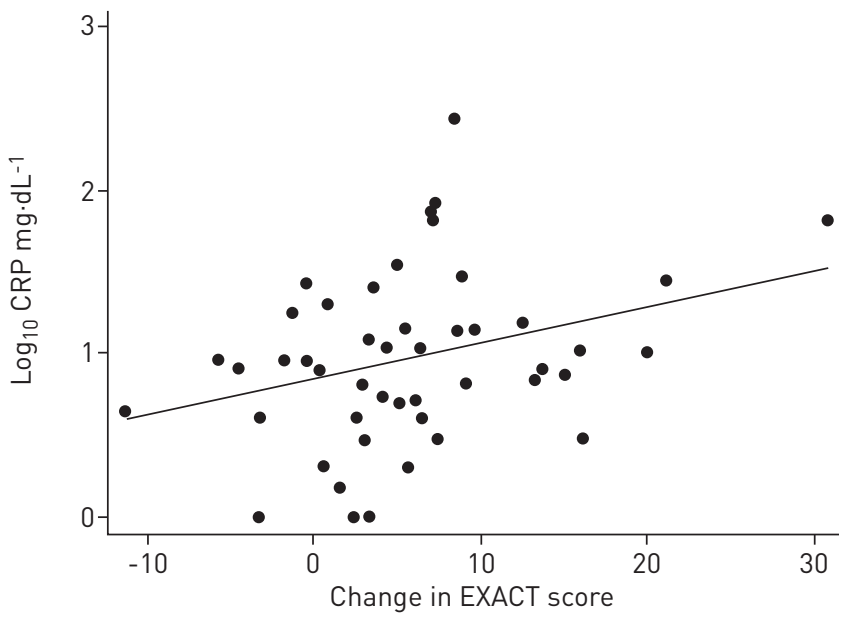

cohort diary card symptom counts and PEFR generated using daily mean values of all 128 exacerbations are presented in figure $3 \mathrm{a}-\mathrm{c}$, respectively.

Relationship between EXACT scores and exacerbation treatment

No difference was observed in exacerbation onset EXACT scores between treated (systemic therapy) and untreated (no systemic therapy) events $(n=85$, mean \pm SD $48.2 \pm 8.5$ versus $n=43,47.7 \pm 9.0 ; \mathrm{p}=0.762$ ). However, maximum EXACT scores were significantly higher in treated than untreated events $(n=85$, mean \pm SD $55.2 \pm 9.1$ versus $\mathrm{n}=43,51.8 \pm 8.1 ; \mathrm{p}=0.040$ ) (fig. $4 \mathrm{a}$ ).

Time course of EXACT scores during treated and untreated exacerbations

Median (interquartile range) time from exacerbation onset to initiation of systemic therapy for the 85 treated exacerbations was 2 days (1-4 days). Despite the higher maximum EXACT score following exacerbation onset, no significant difference was seen between treated and untreated exacerbations in EXACT recovery time $(\mathrm{n}=61$, median 7 days $(0-12$ days $)$ versus $\mathrm{n}=32,5.5$ days $(2-12$ days $) ; \mathrm{p}=0.656)$ (fig. $4 \mathrm{~b})$ or total net EXACT recovery score $(\mathrm{n}=61$, median $24.2 \mathrm{EXACT} \cdot$ days $(0-75.3 \mathrm{EXACT} \cdot$ day) versus $\mathrm{n}=32,13.1 \mathrm{EXACT} \cdot$ day $(1.5-65.9 \mathrm{EXACT} \cdot$ day); $\mathrm{p}=0.455)$, where EXACT·day is the unit for the total net EXACT recovery score of an exacerbation (calculated as the sum of daily mean change from baseline EXACT scores from exacerbation onset to symptomatic resolution as judged by London COPD cohort diary cards).

\section{EXACT concordance}

Relationship between EXACT scores and London COPD cohort diary card-defined exacerbations 58 patients had 128 London COPD cohort diary card-defined exacerbations, 27 of which $(21.1 \%)$ attained a 12-point increase in EXACT score above baseline for two consecutive days. 34 (26.6\%) out of 128 of exacerbations attained a nine-point increase for three consecutive days. 57 (44.5\%) out of 128 exacerbations attained a 12-point increase in EXACT score above baseline at least once during the 14-day period following exacerbation onset. On average this threshold score of 12 was breached 4.4 days after symptomatic onset. 59 (46.1\%) out of 128 exacerbations had a nine-point increase in EXACT score above baseline at least once during the 14-day period following exacerbation onset, on average 3.7 days after symptomatic onset.

\section{Relationship between EXACT scores and HCU exacerbations}

$85(66.4 \%)$ out of 128 London COPD cohort diary card-defined exacerbations were treated with additional systemic therapy (oral antibiotics and/or corticosteroids) by the study team, consistent with a moderateseverity HCU exacerbation. $22(25.9 \%)$ out of $85 \mathrm{HCU}$ exacerbations attained a 12-point increase in EXACT score above baseline for two consecutive days. 29 (34.1\%) of 85 attained a nine-point increase for three consecutive days (see online supplementary material for further threshold analysis). The 12-point threshold was breached at least once during the 14-day period following exacerbation onset in $43(50.6 \%)$ out of 85 of these HCU exacerbations and the nine-point threshold in 44 (51.8\%) out of 85 .

Relationship between symptom-defined events using the EXACT and London COPD cohort diary card and HCU exacerbations

The scoring algorithm for a symptom-defined event using the EXACT is an increase of 12 points above the patient's mean baseline for two consecutive days or an increase of nine points above the patient's mean 

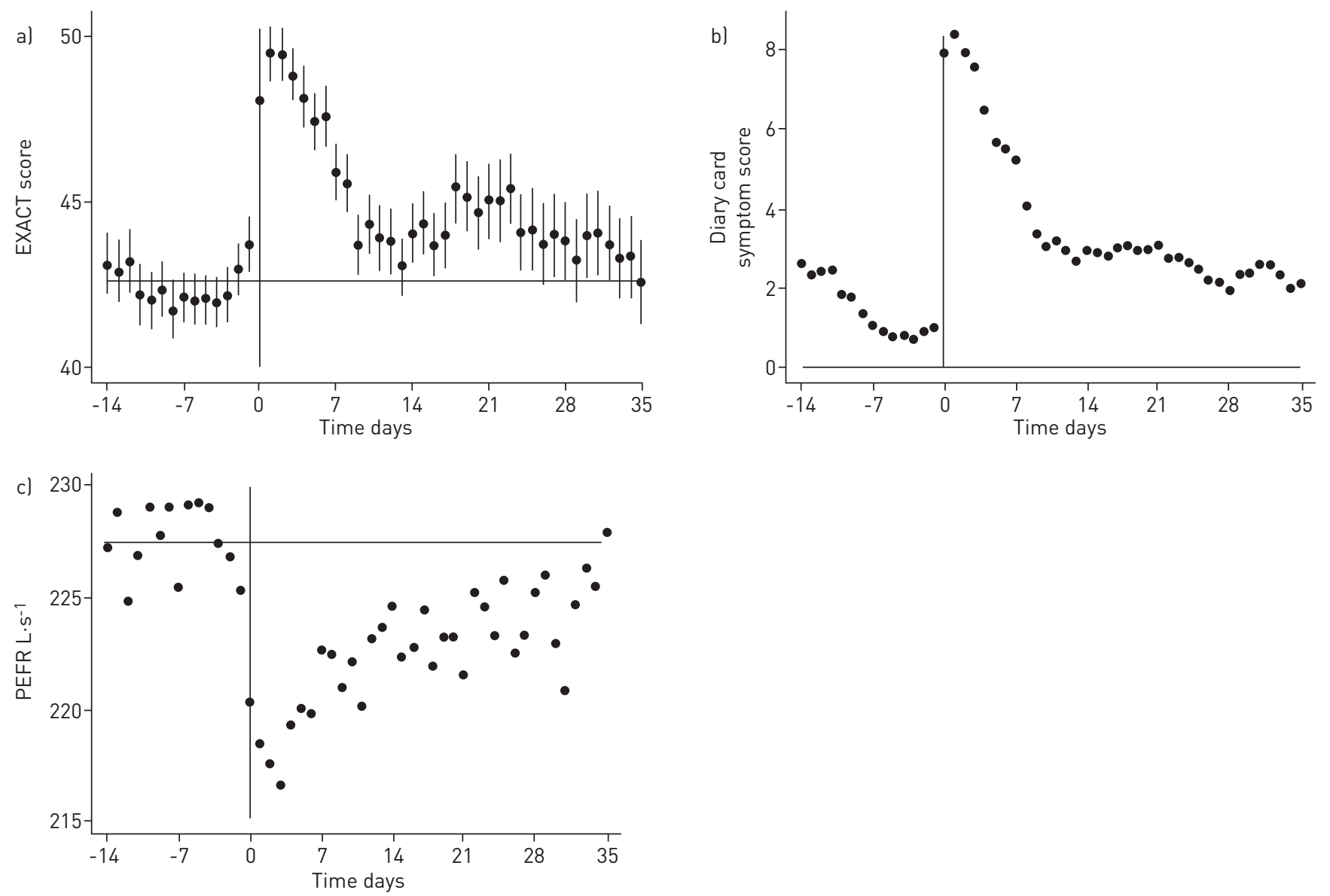

FIGURE 3 Time course plots of recovery of a) exacerbations of chronic pulmonary disease tool (EXACT) score, b) London chronic obstructive pulmonary disease (COPD) cohort diary card symptom counts and c) peak expiratory flow rate (PEFR) generated using daily mean values of all 128 exacerbations. Vertical bars represent standard errors. Horizontal lines indicate mean baseline scores.

baseline for three consecutive days. Previous analyses using the EXACT have examined the day of presentation of an HCU exacerbation \pm 7 days for the presence of an EXACT event (N.K. Leidy and L.T. Murray, United BioSource Corporation (UBC), Bethesda, MD, USA; personal communication). In this study, we analysed the period of exacerbation onset \pm 7 days, as defined by London COPD cohort diary cards. 86
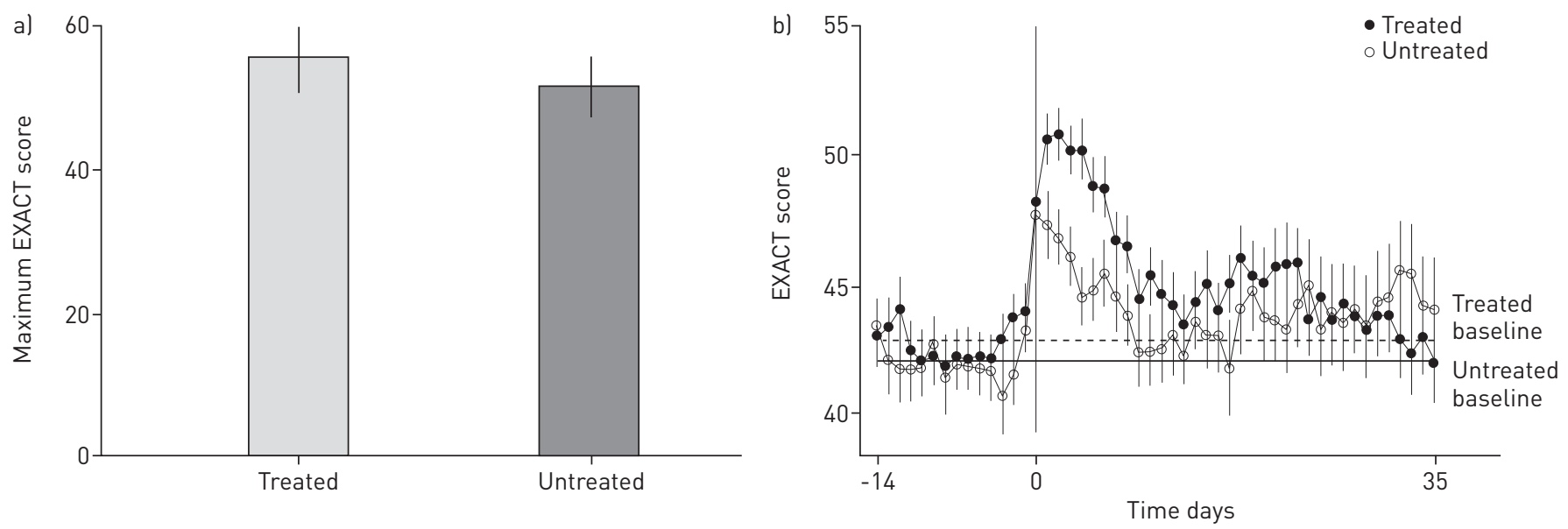

FIGURE 4 a) Maximum exacerbations of chronic pulmonary disease tool (EXACT) scores in chronic obstructive pulmonary disease patients treated and not treated with increased systemic therapy at exacerbation. Vertical lines represent standard errors. b) Time course of EXACT scores during treated and untreated exacerbations. Vertical lines represent standard errors. 
diary card-defined exacerbations had a complete dataset of daily EXACT scores during this period. 24 (27.9\%) of these exacerbations met the EXACT threshold for a symptom-defined event. Of these 86 exacerbations with a complete dataset, 52 were HCU exacerbations, 18 (34.6\%) of which met the criteria for an EXACT event.

Relationship between exacerbation EXACT scores and baseline disease severity

Patients exhibited smaller rises in EXACT score at exacerbation as baseline disease severity (judged by baseline EXACT score) increased. Figure 5 shows that change between maximum exacerbation and baseline EXACT score seen during London COPD cohort diary card exacerbation was significantly related to baseline EXACT score $(\rho=-0.61 ; p<0.001)$ allowing for repeated measures.

\section{Discussion}

This novel study is the first to independently validate the EXACT as an effective tool to assess exacerbation severity. EXACT scores increase at COPD exacerbation, the magnitude of which reflects the severity of the event in terms of treatment, systemic inflammation, airflow limitation and symptom recovery time. For the first time, we have studied the EXACT against the validated London COPD cohort daily symptom diary card $[4,6,12]$, and thus uniquely examined the complex relationship between symptom-defined events using the EXACT and both HCU exacerbations and London COPD cohort diary card-identified, untreated exacerbations. $\sim 50 \%$ of both diary card-defined and HCU exacerbations crossed the EXACT event threshold, but only approximately one-third fulfilled the criteria for an EXACT event. Thus, this study has highlighted important potential limitations of the EXACT in its ability to independently identify events that were captured by physician review (HCU) or London COPD cohort diary cards. Baseline disease severity appears to play an important role in symptom reporting and physician prescribing thresholds at exacerbation.

In this study, we have shown that EXACT scores at exacerbation are significantly related to systemic inflammation, as measured by concurrent levels of serum CRP. This is an important asset for a successful outcome measure of exacerbation severity, as elevated systemic inflammation during exacerbations relates to both clinical nonrecovery and exacerbation recurrence [17]. Furthermore, the time taken for EXACT scores to return to baseline was significantly related to recovery time, as judged by London COPD cohort symptom diary cards, and was also modestly correlated with lung function recovery, as measured by PEFR. Thus, our data support the use of the EXACT as an effective tool to measure exacerbation severity and assess recovery; it is particularly suited to trials of interventions to treat acute exacerbations. In this setting, the relative proportion of patients whose EXACT scores have returned to baseline (or fallen by a predetermined magnitude) by 7 days (the median recovery time in a study evaluating recovery in $>500$ exacerbations [12]) could be a valuable outcome measure.

In this article, patients prospectively completed both London COPD cohort diary cards and EXACT questionnaires to allow us to examine EXACT changes during unreported exacerbations that were not treated with increased systemic therapy. EXACT scores recorded on the day of exacerbation onset were the same for both reported and unreported events. However, while no significant difference was seen in total symptomatic burden or exacerbation length, as judged by EXACT scores between either type of exacerbation, the pattern of recovery observed was different, and maximum EXACT scores recorded during the exacerbation were significantly higher in treated compared with untreated, unreported events. Furthermore, the lack of statistical significance in the total net EXACT recovery score may be due to insufficient sample size. These results demonstrate for the first time that the EXACT is responsive to both reported HCU exacerbations and unreported exacerbations where patients do not report symptomatic deterioration to primary care physicians or members of a research team. Identification of unreported exacerbations may be a particular advantage in pharmaceutical interventional trials, which historically have experienced unexpectedly low rates of HCU exacerbations [18, 19].

The assessment of exacerbation frequency during clinical trials was an initial aim in the development of the EXACT $[14,20]$. To facilitate the identification of unreported exacerbations, the instrument pre-specifies a threshold for these symptom-defined events based on a persistent increase in EXACT score over 2-3 days. In this study, we investigated the relationship between exacerbations identified using the HCU definition, the London Cohort diary card and the EXACT. We found the strength of the relationship to be modest. It should be noted that patients in the London COPD cohort are instructed to report increased respiratory symptoms as recorded in their diary to ensure prompt assessment and therapy as required, and were not instructed to report based upon EXACT responses. Some studies have attempted to use EXACT scores to enhance reporting of exacerbations by remotely monitoring EXACT scores in real time and using worsening scores to generate an alert regarding a possible exacerbation [21]; however, this was not the case in our study.

Both the London COPD cohort diary cards and the EXACT are responsive to a worsening of respiratory symptoms. However, the EXACT requires the increase in symptoms to meet a strict numerical threshold to 


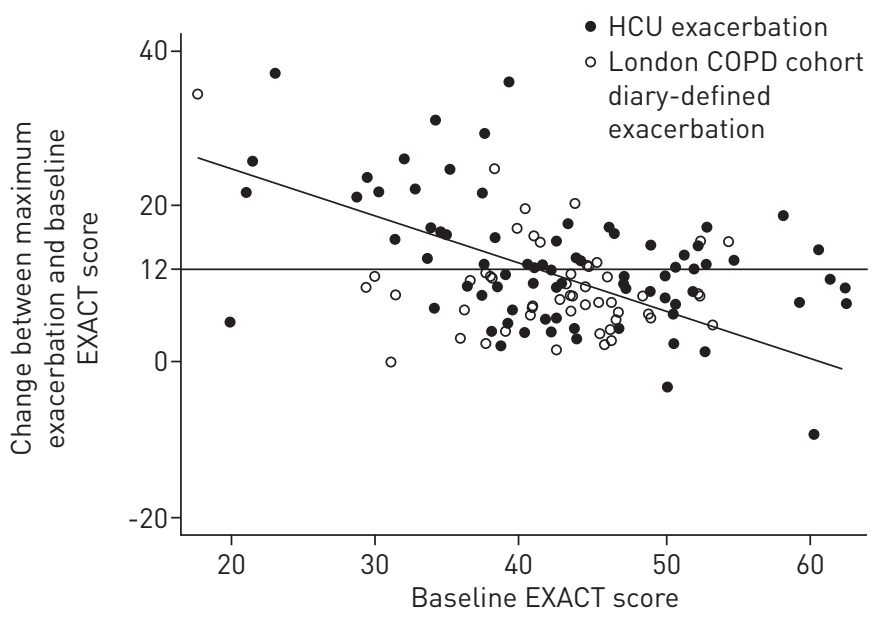

FIGURE 5 Relationship between baseline exacerbations of chronic pulmonary disease tool (EXACT) score and maximum rise in EXACT score at exacerbation. Open circles represent London chronic obstructive pulmonary disease (COPD) cohort diary card-defined exacerbations which were not treated with additional systemic therapy.

fulfil criteria for symptom-defined events using the EXACT, thus, potentially increasing the likelihood of undercounting relative to London COPD cohort diary card-defined exacerbations. Furthermore, the seminal event for the calculation of concordance of EXACT symptom-defined events is more typically the HCU exacerbation, with the date of treatment administration as the day of onset, unlike in this article where we have used cohort diary cards to define the onset of events and then examined the corresponding EXACT scores of both HCU and London COPD cohort diary card-defined exacerbations.

Patients in the London COPD cohort are specifically trained to rapidly recognise and report the increased respiratory symptoms that characterise COPD exacerbations. This may also have impacted on the relationship between symptom-defined events using the EXACT and HCU exacerbations seen in the clinic. As our patients present early in the course of their exacerbation and receive prompt systemic treatment, this may alter the evolution of symptoms for HCU exacerbations compared with when presentation is markedly delayed. In such circumstances, if patients do not commence therapy until late in their exacerbation, their symptoms may be of a higher intensity and, thus, be more likely to cross the threshold for symptom-defined events using the EXACT. Nonetheless, despite these caveats, it remains a concern that only approximately one-third of HCU exacerbations within this study fulfilled the criteria for an EXACT event. The strength of the relationship between EXACT events and HCU exacerbations in our study is consistent with preliminary data from the FORWARD (Foster 48-week Trial to Reduce Exacerbations in COPD) trial, which used the traditional physician diagnosis of exacerbations as a co-primary outcome, and the EXACT to enhance exacerbation detection, and also found marked inconsistencies between EXACT events and HCU exacerbations $[21,22]$.

We examined the relationship between EXACT scores and HCU exacerbations further by exploring the role of baseline COPD disease severity. At baseline, EXACT scores accurately reflected disease severity as judged by lung function impairment and London COPD cohort diary card exacerbation frequency. At exacerbation, patients exhibited smaller rises in EXACT score as baseline disease severity increased, suggesting that patients with more severe stable disease are more likely to report and receive additional systemic therapy at exacerbations associated with smaller increases in symptom intensity than patients with milder baseline disease. This result confirms that people seek care and are treated for exacerbations for a variety of reasons, including varied tolerance to symptomatic change. More severe patients may be more sensitive to change in symptoms, more frightened by smaller changes, or be better trained and, therefore, report their changes earlier. The London COPD cohort is carefully trained to detect change and seek treatment early, and these results support the success of this programme. These results also suggest that the EXACT thresholds for symptom-defined events are conservative, and are not overestimating the frequency of symptom-defined events that are unreported. It remains important to count HCU events as HCU events, particularly as they remain prominent in major guidelines for the diagnosis of an exacerbation [23]. However, all unreported and untreated symptom-defined events also need to be accurately detected with a diary, as these events are both common $[6,9,10]$ and important, contributing to impaired health status $[6,9]$.

The EXACT has previously been used in conjunction with a personal digital assistant $[14,24]$ or smartphone [25]. A potential limitation of our study is that the EXACT was administered in paper rather than electronic format. However, we have previously confirmed that PROs can be reliably completed at exacerbation and during recovery in paper format [26]. Furthermore, because the content of the EXACT is identical in both 
formats, this is unlikely to significantly alter the results obtained. The use of a paper version of the tool also ensures that the results of the study are applicable to the widest range of COPD patients, as patients were not excluded because they were not technically capable of using an electronic instrument.

We have demonstrated that the EXACT is an effective tool to evaluate exacerbation severity in outpatient settings when the event was captured by physician review (HCU) or by London COPD cohort diary cards. Future studies should also assess the efficacy of the instrument in hospitalised patients and can use the EXACT to assess both the maximum symptomatic intensity of exacerbations and the total symptomatic burden of events.

In conclusion, the EXACT is an effective method of evaluating exacerbation severity. EXACT scores reflect severity as determined by lung function, exacerbation length and systemic inflammation. The tool is responsive to both treated and untreated exacerbations and can be effectively used in conjunction with daily symptom diary cards to provide novel outcome measures in clinical trials of acute exacerbation therapies. However, uncertainty remains regarding the effectiveness of the instrument to independently and accurately detect the onset and frequency of exacerbations, a particular concern in the study of preventative therapies for COPD exacerbations.

\section{Acknowledgements}

The authors would like to thank N.K. Leidy, L. Murray and colleagues at the United BioSource Company (Bethesda, MD, USA) for the use of the EXACT in this study.

\section{References}

1 Wedzicha JA, Seemungal TA. COPD exacerbations: defining their cause and prevention. Lancet 2007; 370: 786-796.

2 British Thoracic Society. Burden of Lung Disease Report, 2006. www.brit-thoracic.org.uk/Delivery-of-RespiratoryCare/Burden-of-Lung-Disease-Reports.aspx Date last accessed: October 15, 2013.

3 Hurst JR, Vestbo J, Anzueto A, et al. Susceptibility to exacerbation in chronic obstructive pulmonary disease. N Engl J Med 2010; 363: 1128-1138.

4 Donaldson GC, Seemungal TA, Bhowmik A, et al. Relationship between exacerbation frequency and lung function decline in chronic obstructive pulmonary disease. Thorax 2002; 57: 847-852.

5 Kanner RE, Anthonisen NR, Connett JE. Lower respiratory illnesses promote FEV1 decline in current smokers but not ex-smokers with mild chronic obstructive pulmonary disease: results from the Lung Health Study. Am J Respir Crit Care Med 2001; 164: 358-364.

6 Seemungal TA, Donaldson GC, Paul EA, et al. Effect of exacerbation on quality of life in patients with chronic obstructive pulmonary disease. Am J Respir Crit Care Med 1998; 157: 1418-1422.

7 Donaldson GC, Hurst JR, Smith CJ, et al. Increased risk of myocardial infarction and stroke following exacerbation of COPD. Chest 2010; 137: 1091-1097.

8 Soler-Cataluña JJ, Martínez-García MA, Román Sánchez P, et al. Severe acute exacerbations and mortality in patients with chronic obstructive pulmonary disease. Thorax 2005; 60: 925-931.

9 Langsetmo L, Platt RW, Ernst P, et al. Underreporting exacerbation of chronic obstructive pulmonary disease in a longitudinal cohort. Am J Respir Crit Care Med 2008; 177: 396-401.

$10 \mathrm{Xu} \mathrm{W}$, Collet JP, Shapiro S, et al. Negative impacts of unreported COPD exacerbations on health-related quality of life at 1 year. Eur Respir J 2010; 35: 1022-1030.

11 Wilkinson TM, Donaldson GC, Hurst JR, et al. Early therapy improves outcomes of exacerbations of chronic obstructive pulmonary disease. Am J Respir Crit Care Med 2004; 169: 1298-1303.

12 Seemungal TA, Donaldson GC, Bhowmik A, et al. Time course and recovery of exacerbations in patients with chronic obstructive pulmonary disease. Am J Respir Crit Care Med 2000; 161: 1608-1613.

13 Food and Drug Administration. Guidance for industry on patient-reported outcome measures: use in medical product development to support labeling claims. Federal Register 2009; 74: 65132-65133.

14 Leidy NK, Wilcox TK, Jones PW, et al. Standardizing measurement of chronic obstructive pulmonary disease exacerbations. Reliability and validity of a patient-reported diary. Am J Respir Crit Care Med 2011; 183: 323-329.

15 Quanjer PH, Tammeling GJ, Cotes JE, et al. Lung volumes and forced ventilatory flows. Report Working Party Standardization of Lung Function Tests, European Community for Steel and Coal. Official Statement of the European Respiratory Society. Eur Respir J 1993; 6: Suppl. 16, 5-40.

16 Quint JK, Donaldson GC, Hurst JR, et al. Predictive accuracy of patient-reported exacerbation frequency in COPD. Eur Respir J 2011; 37: 501-507.

17 Perera WR, Hurst JR, Wilkinson TM, et al. Inflammatory changes, recovery and recurrence at COPD exacerbation. Eur Respir J 2007; 29: 527-534.

18 Wedzicha JA, Calverley PM, Seemungal TA, et al. The prevention of chronic obstructive pulmonary disease exacerbations by salmeterol/fluticasone propionate or tiotropium bromide. Am J Respir Crit Care Med 2008; 177: $19-26$.

19 Calverley PM, Sanchez-Toril F, McIvor A, et al. Effect of 1-year treatment with roflumilast in severe chronic obstructive pulmonary disease. Am J Respir Crit Care Med 2007; 176: 154-161.

20 Leidy NK, Wilcox TK, Jones PW, et al. Development of the EXAcerbations of Chronic Obstructive Pulmonary Disease Tool (EXACT): a patient-reported outcome (PRO) measure. Value Health 2010; 13: 965-975.

21 Singh D, Kampschulte J, Wedzicha JA, et al. A trial of beclomethasone/formoterol in COPD using EXACT-PRO to measure exacerbations. Eur Respir J 2013; 41: 12-17. 
22 Wedzicha JA, Agusti A, Vestbo J, et al. Novel detection of exacerbations of COPD with patient reported outcome (EXACT-PRO) and Blackberry (BB). Eur Respir J 2010; 36: Suppl. 54, 43s.

23 Global Initiative for Chronic Obstructive Lung Disease. Global strategy for the diagnosis, management and prevention of COPD. Global initiative for chronic Obstructive Lung Disease (GOLD) 2013. www.goldcopd.org/ guidelines-global-strategy-for-diagnosis-management.html Date last updated: February 2013. Date last accessed: October 15, 2013.

24 Jones PW, Chen WH, Wilcox TK, et al. Characterizing and quantifying the symptomatic features of COPD exacerbations. Chest 2011; 139: 1388-1394.

25 Halpin DM, Laing-Morton T, Spedding S, et al. A randomised controlled trial of the effect of automated interactive calling combined with a health risk forecast on frequency and severity of exacerbations of COPD assessed clinically and using EXACT PRO. Prim Care Respir J 2011; 20: 324-331.

26 Mackay AJ, Donaldson GC, Patel AR, et al. Usefulness of the chronic obstructive pulmonary disease assessment test to evaluate severity of COPD exacerbations. Am J Respir Crit Care Med 2012; 185: 1218-1224. 